\title{
Carbidopa/levodopa/entacapone: the evidence for its place in the treatment of Parkinson's disease
}

This article was published in the following Dove Press journal:

Core Evidence

18 December 2009

Number of times this article has been viewed

\section{Markos Poulopoulos Cheryl Waters}

Department of Neurology, Division of Movement Disorders, Columbia University Medical Center, Neurological Institute of New York, Center of Parkinson's Disease and Other Movement Disorders, New York, NY, USA
Correspondence: Markos Poulopoulos Neurological Institute of New York, 710 west I68th street, New York, NY 10032, USA

$\mathrm{Tel}+\mathrm{I} 2 \mathrm{I} 23050202$

Fax +I 212305 I304

Emailmp2917@columbia.edu
Introduction: Parkinson's disease (PD) is a common neurodegenerative disease. In the 1960s, it was shown that the degeneration of dopamine producing neurons in the substantia nigra (SN) caused the motor features of PD. Dopamine replacement with levodopa, a dopamine precursor, resulted in remarkable benefit. Yet, the intermittent administration of levodopa is a major cause of motor complications, such as "wearing-off" of levodopa's benefit and involuntary movements, known as dyskinesia. Therefore, agents that prolong levodopa's half-life were employed, such as carbidopa, an aromatic amino acid decarboxylase (AADC) inhibitor, and entacapone, a catecholO-methyltransferase (COMT) inhibitor. The combination product carbidopa/levodopa/entacapone (CLE) was approved in 2003 for the treatment of PD patients.

Aims: To assess the evidence for the place of CLE in the treatment of PD.

Evidence review: CLE has a good efficacy, safety and tolerability profile, similar to that of entacapone taken separately with carbidopa/levodopa (CL). Compared to CL alone, it prolongs levodopa's benefit, and improves the quality of life but not the motor performance in PD patients with nondebilitating "wearing-off" or dyskinesia. However, it increases the dyskinesia rate in early PD patients, and has adverse events in advanced patients with significant motor complications. There is insufficient evidence regarding cost-effectiveness.

Place in therapy: CLE is an attractive alternative for patients with nondisabling "wearing-off" or dyskinesia taking CL with or without entacapone. It cannot be recommended for early PD patients, as it can induce more dyskinesia than CL alone, or in any patients who seem to have more adverse events.

Keywords: Parkinson's disease, levodopa, entacapone, carbidopa, treatment

Core evidence place in therapy summary for carbidopa/levodopa/entacapone

\begin{tabular}{|c|c|c|}
\hline Outcome measure & Evidence & Implications \\
\hline \multicolumn{3}{|l|}{ Disease-oriented evidence } \\
\hline UPDRS (part II, III, and II+III) & Consistent & $\begin{array}{l}\text { Part II improved when switched from CL to CLE } \\
\text { in patient with wearing-off with or without mild } \\
\text { dyskinesia. Also improved when CLE compared } \\
\text { to CL, in CL naïve early PD patients. Part III } \\
\text { scores were not different. }\end{array}$ \\
\hline $\begin{array}{l}\text { "Off" time (UPDRS } \\
\text { question 39) }\end{array}$ & Clear & $\begin{array}{l}\text { Improved significantly when switched from CL to } \\
\text { CLE and when compared directly in early PD. }\end{array}$ \\
\hline Dyskinesia & $\begin{array}{l}\text { Mostly } \\
\text { negative }\end{array}$ & $\begin{array}{l}\text { STRIDE-PD data showed higher rates } \\
\text { of dyskinesia with shorter time to dyskinesia } \\
\text { in patients treated with CLE. }\end{array}$ \\
\hline
\end{tabular}

submit your manuscript | www.dovepress.com

Dovepress 


\begin{tabular}{|c|c|c|}
\hline \multicolumn{3}{|l|}{ (Continued) } \\
\hline Outcome measure & Evidence & Implications \\
\hline PDQ-39 & Ambiguous & $\begin{array}{l}\text { Improved in patients taking CLE after switching } \\
\text { from CL with moderate disease, but there was no } \\
\text { change in direct comparison in the early stages. }\end{array}$ \\
\hline PDQ-8 & Clear & $\begin{array}{l}\text { Better outcome in patients treated with CLE at an } \\
\text { early stage of the disease. }\end{array}$ \\
\hline CGI-C (investigator) & Clear & $\begin{array}{l}\text { Equivalent improvement in both CLE, and } \\
\text { carbidopa/levodopa and entacapone when } \\
\text { compared with CL only, in mild to moderate } \\
\text { disease severity. }\end{array}$ \\
\hline \multicolumn{3}{|c|}{ Patient-oriented evidence } \\
\hline Discontinuation rate & Clear & $\begin{array}{l}\text { Acceptable and equal to entacapone studies. } \\
\text { Higher rate in older patients with more than } \\
10 \text { years disease duration. }\end{array}$ \\
\hline Adverse events & Clear & $\begin{array}{l}\text { Expected at the rate of the previous entacapone } \\
\text { studies. Rated as mild to moderate by the patients. }\end{array}$ \\
\hline PDQ-39 & Ambiguous & $\begin{array}{l}\text { Improved in patients taking CLE after switching } \\
\text { from } C L \text { with moderate disease, but there was } \\
\text { no change in direct comparison in early disease } \\
\text { stages. }\end{array}$ \\
\hline QOL-VAS & Clear & Improved when switched from CL to CLE. \\
\hline UPDRS part II & Clear & $\begin{array}{l}\text { Improved quality of life in CLE treated patients } \\
\text { with mild disease severity. }\end{array}$ \\
\hline CGI-C (patient) & Clear & $\begin{array}{l}\text { Equivalent improvement in both CLE and } \\
\text { carbidopa/levodopa and entacapone when } \\
\text { compared with CL only, in mild to moderate } \\
\text { disease severity. }\end{array}$ \\
\hline Patient preference & Clear & $\begin{array}{l}\text { Majority of patients preferred CLE over carbi- } \\
\text { dopa/levodopa and entacapone taken separately } \\
\text { mainly due to convenience, less pill burden, better } \\
\text { compliance and ease to swallow. }\end{array}$ \\
\hline \multicolumn{3}{|l|}{ Economic evidence } \\
\hline & $\begin{array}{l}\text { Insufficient } \\
\text { evidence }\end{array}$ & $\begin{array}{l}\text { One study from the UK found favorable } \\
\text { cost-effectiveness for CLE compared with CL } \\
\text { treatment in PD patients with wearing-off. It is } \\
\text { difficult to generalize this conclusion to different } \\
\text { health-economic systems. }\end{array}$ \\
\hline \multicolumn{3}{|c|}{$\begin{array}{l}\text { Abbreviations: PDQ-39, Parkinson's Disease Questionnaire-39 (quality of life assessment tool); PDQ-8: Parkinson's } \\
\text { Disease Questionnaire-8 (quality of life); UPDRS, Unified Parkinson's Disease Rating Scale (part II scores activities of } \\
\text { daily leaving and part III objective motor performance); CGI-C, Clinical Global Impression of Change; QOL-VAS: Quality } \\
\text { Of Life-Visual Analog Scale; CL: carbidopa/levodopa; CLE, carbidopa/levodopa/entacapone. }\end{array}$} \\
\hline
\end{tabular}

\section{Scope, aims, and objectives}

The combination product of levodopa, carbidopa and entacapone is known generically as "carbidopa/levodopa/ entacapone", but will be referred to here as CLE. Levodopa, an aromatic amino acid, is the most efficacious treatment of Parkinson's disease (PD) that was developed in the late 1960s. ${ }^{1}$ It is the precursor of dopamine and crosses the bloodbrain barrier, unlike dopamine. However, only $1 \%$ of an oral levodopa dose reaches the brain. Approximately $70 \%$ of oral levodopa is metabolized by aromatic amino acid decarboxylase (AADC) in the intestinal mucosa and liver. That leads to increased dopamine levels in the periphery resulting in side effects, namely postural hypotension, nausea and vomiting. ${ }^{2}$ Carbidopa is an inhibitor of peripheral AADC. Its addition to levodopa since the 1970s has decreased the incidence of the aforementioned side effects and increased the availability of levodopa to the brain. It increases the half-life of levodopa from 60 to 90 minutes, thus allowing for a 90\% levodopa dose reduction to achieve the same benefit. ${ }^{3}$ Carbidopa is used in the USA and benserazide or carbidopa in Europe. However, this strategy shifts the peripheral metabolism of levodopa to another pathway. Catechol-O-methyltransferase (COMT) 
transfers the methyl group of S-adenosyl-L-methionine to levodopa producing 3-O-methyldopa (3-OMD), an inactive compound at the periphery, and 3-methoxytyramine (3-MT) in the brain. ${ }^{2,4}$ Entacapone is a peripheral COMT inhibitor. Its pharmacokinetics are unaltered by concomitant administration of levodopa/AADC inhibitor, age, gender, race or multiple dosing (no accumulation has been observed with up to 10 times daily dosing). ${ }^{4}$ Its time to peak concentration $\left(\mathrm{t}_{\max }\right)$ is 2 hours with a half-life of 0.4 to 0.9 hours, both similar to levodopa. It inhibits by $65 \%$ the COMT activity in human erythrocytes after a dose of $200 \mathrm{mg}$. Its bioavailability is between $29 \%$ and $46 \%$ and is albumin bound by $98 \%$. When given together with a dose of levodopa/ carbidopa, it increases both levodopa's half-life and its area under the curve (AUC) by up to $85 \%$ and $50 \%$, respectively. At the same time, it does not affect the peak concentration $\left(\mathrm{C}_{\max }\right)$ and $\mathrm{t}_{\max }$ of levodopa. ${ }^{4-6}$ Finally, it does not interact with compounds that are metabolized by COMT, such as epinephrine, isoproterenol, alpha-methyldopa, dopamine, dobutamide, AADC inhibitors and nadolol, or increase the (nor)epinephrine plasma levels when used concomitantly with imipramine or monoamine oxidase (MAO) A inhibitors. ${ }^{4}$ The clinical efficacy of entacapone was observed in prospective, randomized, double-blind studies on PD patients with motor fluctuations (levodopa positive effect "wears-off" typically before the next dose). Ruottinen and Rinne observed that the addition of $200 \mathrm{mg}$ of entacapone to each dose of levodopa/ carbidopa increased the "on" time (when patient experiences benefit from levodopa) by 2.5 hours and reduced by $16 \%$ the daily levodopa dose. ${ }^{6}$ In the SEESAW study, entacapone increased daily "on" time by 1 hour, decreased the daily levodopa dose by $12 \%$ and improved activities of daily living (ADL), motor and total scores of the Unified Parkinson's Disease Rating Scale (UPDRS). ${ }^{7}$ In the NOMECOMT study, the daily "on" time increased by 1.4 hours with levodopa daily dose reduction of $12 \% .{ }^{8}$ This effect was sustained in a 3-year open label extension of the study. In the CELOMEN study, the daily "on" time increased by 1.7 hours, the daily "off" time decreased by 1.5 hours, the mean daily levodopa dose was reduced by $54 \mathrm{mg}$, and the ADL, motor and total UPDRS scores improved. ${ }^{9}$ In the UK-IRISH study, the daily "on" time increased by 1.3 hours and the "off" time decreased by 1.1 hours. ${ }^{10}$

These results coupled with bioequivalence studies lead to CLE approval by the US Food and Drug Administration (FDA) in 2003 for substitution of carbidopa/levodopa (CL) and entacapone taken separately and for replacing CL in PD patients experiencing "wearing-off" signs and symptoms (for patients taking daily levodopa dose up to $600 \mathrm{mg}$ and not experiencing dyskinesia). The aim of this article is to review the evidence for safety, tolerability, efficacy and cost-effectiveness of CLE and its place in the treatment of PD.

\section{Methods}

We reviewed the literature by using the PubMed, Medline and Cochrane databases. We used the search terms "Stalevo" and "entacapone". The search produced 21 articles about CLE in PubMed and Medline combined and zero articles in Cochrane database. Of these, 8 articles were foreign, could not be obtained, and were excluded. Of the remaining, 9 articles were original papers, 3 were review articles and 1 was an abstract about cost-effectiveness. We included unpublished, to this point, data from the STRIDE-PD study.

\section{Disease overview}

Parkinson's disease is named after James Parkinson who published in 1817 a monograph with the title "An Essay on the Shaking Palsy". ${ }^{11}$ The incidence of PD is about 14 per 100,000 population per year overall but increases to 160 for the population aged 70 years or older. The median prevalence is 9.4 per 1000 with a $\%$ risk for developing PD by the age of 80 . It affects approximately 1 million individuals in the USA. ${ }^{12,13}$ The mortality rate was reported to be 3-fold greater in PD patients before the introduction of levodopa. This was reduced to 1.6-fold in the post-levodopa era with a trend to increase again. ${ }^{14,15}$ The pathologic hallmark of PD is the loss of dopamine producing neurons in the substantia nigra $(\mathrm{SN})$ accompanied by the intraneuronal accumulation of alpha-synuclein containing Lewy bodies (LB) resulting in dopamine deficiency. ${ }^{16,17}$ There is much research on the etiology and pathogenesis of PD. Although genetic causes account for only $10 \%$ of the cases, the discovery of gene products and their function gave us better insight as to the possible pathogenic mechanisms. In particular, the concept of protein mishandling seems important, and involves the ubiquitin-proteasomal system and the lysosomes. Mitochondrial dysfunction, oxidative stress, apoptosis, and inflammation are also important mechanisms that possibly interact with each other. ${ }^{18-22}$ Environmental factors can have a harmful (like pesticides), ${ }^{23}$ or protective effect (cigarette smoking) ${ }^{24}$

PD typically has an asymmetric onset with bradykinesia, rest tremor, rigidity and postural instability being its cardinal motor features. ${ }^{22,25}$ Bradykinesia is the most characteristic manifestation of PD and presents with slowness of movements resulting in loss of facial expression (hypomimia), 
low volume soft voice, reduced blinking, drooling and slow gait with poor arm swing. It is due to dopamine deficiency and failure of the positive feedback from the basal ganglia to the frontal cortex. ${ }^{26}$ Rest tremor is a 4 to $6 \mathrm{~Hz}$ oscillatory movement involving the distal parts of the extremities, such as hand ("pill-rolling" tremor) and foot. It is unilateral at onset and more prominent on the side it started when the disease progressively involves the other side. Interestingly, some patients report postural tremor years before the onset of PD. ${ }^{27}$ Rigidity is the resistance during passive movement of the limb, neck, or trunk. It may be associated with pain. Painful shoulder is a common initial manifestation of PD and usually misdiagnosed as of musculoskeletal origin. ${ }^{28}$ Postural instability is the loss of postural reflexes and usually occurs in later stages. It is demonstrated when a patient loses his balance while pulled forward or backward by the shoulders ("pull test"). ${ }^{26}$

In addition to the cardinal features, there are other motor manifestations of PD. Freezing is a sudden and transient loss of movement for a few seconds and typically involves the gait. It is a frequent cause of falls and can be only partially relieved by levodopa therapy. Other features are dysarthria, dysphagia, sialorrhea, decreased ocular convergence, and appearance of primitive reflexes, such as glabellar and palmomental. ${ }^{26}$

Nonmotor manifestations of PD are thought to be derived from neurotransmitter deficits other than dopamine, as opposed to most of the classic motor features. ${ }^{29,30}$ Those are categorized into neuropsychiatric, sleep, autonomic, and sensory symptoms. Neuropsychiatric manifestations include depression, anxiety, apathy, psychosis, and cognitive impairment. Dysautonomia can manifest as orthostatic hypotension, hyperhidrosis, erectile dysfunction, constipation and bladder dysfunction. Finally, sensory symptoms take the form of olfactory dysfunction, akathisia, paresthesia, and pain. ${ }^{26,29,30}$

\section{Current therapy options}

Treatment for PD falls into three categories: therapies, medications and surgery. We will focus on the treatment of the cardinal motor features of PD, the majority of which are dopamine responsive. Physical and other therapies, surgery and the treatment of the nonmotor nondopamine responsive symptoms are beyond the scope of this article; the reader is referred to detailed reviews of these topics. ${ }^{22,31,32}$

The cardinal motor features of PD, bradykinesia and rigidity, respond very well to dopamine replacement treatment. Tremor can be less responsive, whereas postural instability shows the least response to dopaminergic strategies. Levodopa is the most effective medication and has been employed in the treatment of PD since the late 1960s. ${ }^{1,33}$ Currently, we have a variety of dopaminergic and non-dopaminergic medications available. Dopaminergic agents are: dopamine precursor (levodopa), decarboxylase inhibitors (carbidopa, benserazide), dopamine agonists (pramipexole, ropinirole, pergolide, bromocriptine, apomorphine, cabergoline, lisuride), COMT inhibitors (entacapone, tolcapone), type B MAO inhibitors (selegiline, rasagiline) and dopamine releaser/anti-glutamergic (amantadine). Anticholinergics have been used since the 1950s for symptomatic relief, due to their anti-muscarinic effect. However, they result in memory impairment and hallucinations, especially in the elderly. Therefore, they are rarely used. They are less effective than their dopaminergic counterparts. The most significant benefit is on tremor severity. ${ }^{22,31,32}$

Over time, treatment with levodopa is associated with "wearing-off" phenomenon (treatment effect wears off with return of parkinsonian symptoms) and dyskinesia (choreiform involuntary movements and dystonia). The ELLDOPA study showed a dose-response improvement in UPDRS scores, but also a dose-response effect regarding motor complications, with dyskinesia occurring in $16.5 \%$ and wearing-off in $28 \%$ of patients treated with daily levodopa dose of $600 \mathrm{mg}$ for 9 months. ${ }^{34}$ On the other hand, the CALM-PD study compared pramipexole to levodopa with respect to their potential for developing motor complications. Pramipexole-treated patients had less incidence of dyskinesia (10\% versus $31 \%)$ and wearing-off periods $(24 \%$ versus $38 \%$ ) after 2 years. ${ }^{35}$ Pramipexole was less effective and associated with more somnolence than levodopa. Therefore, in early PD patients there is some debate about whether to initiate treatment with levodopa or dopamine agonists. In the treatment of moderate and more advanced disease nonlevodopa medications are not effective and the introduction of levodopa is required. ${ }^{22,31,32}$

\section{Unmet needs}

The major unmet need is the discovery of a neuroprotective treatment. To date, there is no definite evidence for any of the marketed medications to be neuroprotective. A detailed discussion is beyond the scope of this article and the reader is referred to relevant reviews. 22,31

The management of motor complications (wearing-off and dyskinesia) poses significant problems in clinical practice. The pathogenesis of the motor complications is believed to be derived from the pharmacokinetics of the dopaminergic 
medications (especially levodopa) and the reduced dopamine-producing neurons in the substantia nigra (SN). Normally, the SN delivers dopamine to the striatum in a tonic, continuous manner. In PD patients we substitute the deficit in dopamine production with an intermittent pattern of delivery, owing to the short half-life of levodopa and the decreased buffering potential of the remaining functioning SN neurons. This nonphysiologic pattern results in postsynaptic alterations that involve gene transcription and the firing pattern of the basal ganglia output neurons. Therefore, the current trend is to develop strategies that provide continuous dopaminergic stimulation. ${ }^{31}$

\section{Clinical evidence with carbidopa/ levodopa/entacapone}

In the first section we discussed the evidence for the use of entacapone. Entacapone has a similar pharmacokinetic profile to levodopa, and taken together increases levodopa's halflife and bioavailability. This approach leads to increasing the daily "on" time, decreasing the daily "off" time and the daily levodopa dose. ${ }^{6-10}$

A product that combines carbidopa, levodopa, and entacapone in one tablet should have similar pharmacokinetic and efficacy profile. However, the initial step is to prove bioequivalence between CL and entacapone taken separately and combined in one tablet. Four bioequivalence studies tested CLE at different doses (two with CLE containing $50 \mathrm{mg}$ of levodopa, another with 100, and the fourth with $150 \mathrm{mg}$ of levodopa per CLE tablet). There were 40 healthy volunteers per study. They used an open, randomized, crossover and replicate design. They administered either the test drug (CLE) or the reference drug (CL and entacapone) after an overnight fast. Fifteen blood samples were drawn at the same point in time for up to 12 hours after drug administration. Plasma concentrations of levodopa, carbidopa, and entacapone were measured. To determine bioequivalence they calculated the area under the concentration-time curve from 0 up to 12 hours $\left(\mathrm{AUC}_{1-12 \mathrm{~h}}\right), \mathrm{C}_{\max }, \mathrm{t}_{\max }$, and the elimination half-life for levodopa, carbidopa, and entacapone separately. The bioequivalence criteria were met for all three components of all different CLE doses, the levodopa half-life for CLE being 1.7 hours (range 1.2 to 3.1 hours). ${ }^{36}$

\section{Efficacy, safety, and tolerability}

All the CLE studies that assessed efficacy, safety and tolerability will be analyzed. The endpoints for efficacy were the UPDRS, Parkinson's Disease Questionnaire-39 (PDQ-39: including quality of life assessment), Parkinson's Disease
Questionnaire-8 (PDQ-8: quality of life), Clinical Global Impression (CGI for patients and investigators), Quality Of Life-Visual Analog Scale (QOL-VAS). Safety was assessed by the rate of adverse event (AE), and tolerability by the discontinuation rate.

The first study to assess safety and tolerability of CLE in PD patients was conducted by the SELECT-TC Study Group; an open-label, single-arm, 4-week investigation. They recruited PD patients, experiencing wearing-off, with or without mild dyskinesia. All were receiving a stable dose of immediate release formulation CL (25/100) for at least 1 month prior to study entry. Patients were directly switched from immediate release CL to the equivalent dose of CLE. Tolerability was the primary endpoint. The secondary points were percentage with new onset dyskinesia and worsening of pre-existing dyskinesia. Safety assessments included adverse events and measurement of vital signs, EKG, physical examination, and blood and urine values. One hundred and sixty-nine patients were studied. The mean age was 66 years and mean disease duration was 5.23 years. The mean Hoehn and Yahr (H\&Y) stage was 2.28 and 23\% had mild dyskinesia at study entry. Eight percent of patients discontinued the study, the majority due to adverse events, including nausea, worsening of "off" periods, blurred vision, headache, vivid dreams, lightheadedness, and joint pain. Dyskinesia developed in $8.5 \%$ of subjects without history of dyskinesia and it worsened in $43.6 \%$ of those with pre-existing dyskinesia. The majority improved in their dyskinesia with or without reducing the CLE dose. All adverse events were mild and infrequent with nausea being the most frequent (in $12.4 \%$ of patents). No clinical relevant changes in vital signs or laboratory testing were noted. ${ }^{37}$ The conclusion was that the CLE was tolerated by subjects previously treated with immediate release formulation CL $(25 / 100)$.

The TC-INIT study group conducted the first randomized, multinational, parallel-group clinical trial that compared the efficacy and safety of CLE to CL and entacapone in PD patients with end-of-dose wearing-off and a mean levodopa treatment duration of 5 to 6 years. ${ }^{38}$ They randomized 77 patients to CLE and 88 to CL and entacapone. They used three different CLE doses: 50/12.5/200 mg, 100/25/200 mg and 150/37.5/200 $\mathrm{mg}$ (for levodopa/carbidopa/entacapone respectively). In both groups, the mean age of disease onset was 60 years, the mean disease duration was 6 years, the mean levodopa dose was $472 \mathrm{mg}$ in the CL and entacapone and $493 \mathrm{mg}$ in the CLE group and all patients had mild to moderately advanced disease (H\&Y from 1 to 3 ). They all had wearing-off for at least 1 year and were on a stable 
dose of CL only, for at least 6 weeks prior to study entry. The study comprised a 2 -week dose adjustment period, a 6-week treatment period and a 2 -week follow up. Adverse events were reported by $55 \%$ of the total population without significant difference between treatment groups. $75 \%$ of the adverse events were classified by the patients as "mild", $24 \%$ as "moderate" and 1\% as "severe". Nausea was the most common with a trend to be more frequent in the CLE group (14\% versus $9 \%$ ). Influenza-like symptoms were reported only to CLE group (7\%), whereas dyskinesia was more common in the CLE group (7\% versus 3\%). Discontinuation rate was 5\% in the CLE and 3\% in the CL and entacapone group. Eighty percent of the patients responded that they preferred taking CLE compared with two separate tablets. The mean levodopa dose did not change significantly in either group compared with baseline. The primary efficacy variables were the CGI scores, UPDRS change in part III (motor performance), and the total levodopa dose at week 6. In respect to CGI score, $73 \%$ of the patients in the CLE and $76 \%$ in the CL and entacapone group reported improvement. The investigator CGI scores improved in $79 \%$ of patients in both groups. UPDRS part III scores significantly improved in both the CLE and $\mathrm{CL}$ and entacapone groups $(P<001$ and $P=0.0016$ respectively). The QOL-VAS was significantly better in the CLE group compared with the CL and entacapone group. The conclusion of this trial was that both groups were effective and well tolerated.

Another study assessed the conversion from sustained release CL (SR-CL), or SR-CL and entacapone, or SR-CL and standard CL with or without entacapone to CLE. It was an open-label study with one month follow up. The conversion was done overnight. Each SR-CL 50/200 mg (carbidopa/ levodopa, respectively) converted to CLE 37.5/150/200 mg (carbidopa/levodopa/entacapone, respectively) and each SR-CL 25/100 mg to CLE 25/100/200 mg. They recruited $62 \mathrm{PD}$ patients with mean age of 67 years, disease duration of 11 years and daily levodopa dose of $670 \mathrm{mg}$. $80 \%$ of the patients were having wearing-off at baseline and $47 \%$ dyskinesia. 13 out of 62 patients $(21 \%)$ withdrew before the end of the 1-month period due to adverse events. They were older than the CLE group who completed the study (73 versus 66 years) with longer disease duration (12.5 versus 10 years). Withdrawals were due to nausea in $54 \%$, increased "off" time in 38.5\%, and dyskinesia, dizziness and insomnia in $23 \%$. None of these subjects had been previously exposed to entacapone. Forty-nine patients completed the 1-month follow-up. Forty-two preferred CLE and 7 SR-CL. Adverse events were less frequent in the CLE than the SR-LC group.
Twenty-one were taking entacapone at baseline. In these patients, there were significant improvements only in UPDRS motor scores, by 7 points. In the entacapone-naïve patients, there were significant improvements in UPDRS motor scores (by 4 points) and PDQ-39 activities of daily living (ADL) score by 6 points. $78.6 \%$ of the entacapone-naïve patients and $86 \%$ of the total study population preferred CLE over SR-CL. Those who preferred SR-CL were older (68.6 versus 65.7 years) and had longer disease duration (11.7 versus 10.0 years). ${ }^{39}$ The conclusion of this trial was that CLE was preferred by most patients to SR-CL.

The Simcom Study was a single group, open, crossover, multicenter, phase III study that assessed the switch from CL and entacapone to CLE. Fifty-two PD patients were included. Mean age was 61 years, age at onset of PD was 53 years, disease duration was 9 years, duration of levodopa treatment was 8 years, daily levodopa dose was $509 \mathrm{mg}$ and duration of entacapone treatment was 2 years. It consisted of a 4 week control phase (patients continued their usual regimen), a 4-week treatment phase (patients received CLE at equivalent dose) and a 2-week follow up period (patients returned to their baseline treatment). Efficacy parameters were patient preference, UPDRS and QOL-VAS scores. 54\% of the patients preferred CLE compared with 31\% who preferred the previous treatment $(P=0.162) .86 \%$ of all the levodopa doses were directly replaceable. UPDRS scores (part I for cognition and mood, part II for ADL and part III for motor performance) improved from a mean of 35.6 at baseline by 2.5 points $(P<0.01)$. UPDRS part III scores improved from 24 by 1.9 points $(P<0.01)$. The mean daily levodopa dose in the CLE group was lower by $24.6 \mathrm{mg}$ (from $509 \mathrm{mg}$ at baseline). There was no difference in the QOL-VAS score. Three patients $(6 \%)$ discontinued due to adverse events. Seventeen out of 52 patients developed mild to moderated adverse events. Most common were dyskinesia, nausea, diarrhea, and influenza-like symptoms. ${ }^{40}$ The conclusion of this study was that some measures were improved on CLE compared with CL.

The Spanish Stalevo Study Group assessed whether or not the dose of levodopa should be reduced when switching from CL to CLE in patients with wearing-off and without or with mild dyskinesia at baseline in order to avoid emergence or worsening of dyskinesia. It was a multicenter, prospective, single-blind, randomized and clinically controlled 4 week study. Thirty nine patients were randomized to either group A (receiving the same dose of levodopa in the form of CLE) or group B (reducing the dose of levodopa contained in CLE by $15 \%$ to $25 \%$ ). Efficacy endpoints were UPDRS, 
PDQ-39, CGI change and home "on-off" diaries. Mean age was 68 years in group A and 67 in group B. PD duration was 8.5 and 9.4 years, levodopa duration 7.5 and 8.7 years with a daily dose of $566 \mathrm{mg}$ and $670 \mathrm{mg}$ respectively. H\&Y stage was II to III in $75 \%$ of the patients, but more patients with stage IV were in group A. UPDRS total score at baseline was 55 in group A and 58 in B. Seven patients in group A and eight in group B had dyskinesia at baseline. Six patients in group A (35\%) and 5 in group B (22.7\%) experienced mild and transient adverse events. Nausea, dizziness, somnolence, and abdominal pain were the most common. One patient in group A discontinued due to increased dyskinesia. Objective increase of dyskinesia was documented in two patients in each group. Group A patients experienced a significant increase in "on" time of 76 minutes and significant decrease on "off" time of 123 minutes. Group B experienced a modest increase in "on" time of 38 minutes and decrease in "off" time of 32 minutes. The daily "on" with dyskinesia increased by 33 minutes in group A and by 98 minutes in group B. Total UPDRS score improved by 1 point in "on" state and 6 points in "off" state in group A, and by 3 and 5 points respectively in Group B. PDQ-39 and CGI results regarding quality of life did not change significantly, but more than $60 \%$ of patients felt better in both groups. Dyskinesia was not a major problem in this study. The conclusion of this study was that there was no advantage to reducing the dose of levodopa when switching to CLE. ${ }^{41}$

The START-M open 6 week trial assessed the efficacy, safety and tolerability of CLE when PD patients with wearing off switched from CL. Fifty patients were included with a mean age of 66.7 years, mean disease duration of 6.8 years and a mean daily levodopa dose of $540 \mathrm{mg}$. 38\% of patients had H\&Y stage 2 (bilateral parkinsonism without postural instability), $41 \%$ stage 2.5 (bilateral disease and instability with recovery on pull test) and $32 \%$ stage 3 (bilateral disease with instability and no recovery on pull test). Twenty-seven percent of the patients had dyskinesia. The daily levodopa dose in the form of CLE was equivalent to the baseline dose. Assessment tools were the UPDRS score, motor fluctuation questionnaire and CGI scores. By week 6 there was a significant $(P<0.001)$ reduction of the total UPDRS score from 46.5 to 36 points ( $29 \%$ reduction). Subscale reductions (mean) were as follows: part I by 1.1 points $(30 \%)$, part II by 3.6 points $(25 \%)$ and part III by 4.8 points $(24.7 \%)$. Mean daily "off" time reduced by 1.7 hours. Improvement rate based on CGI was 52\% (patient assessment) and 72\% (physician assessment). Fewer than $10 \%$ of patients experienced side effects, mainly nausea, orthostatic dizziness and headache. None of them required intervention. ${ }^{42}$ This open trial showed improvements on many measures when patients switched to CLE.

The QUEST-AP study group addressed the question whether CLE treatment improved quality of life when compared with CL treatment in PD patients without or with minimal, nondisabling motor fluctuations. It was a randomized, double-blind, parallel-group, active-controlled multicenter 12-week trial. Inclusion criteria were PD patients with $H \& Y$ stage of 1 to 2.5 , with 0 to 3 hours of non-disabling daily "off" time and total daily levodopa dose between $300 \mathrm{mg}$ and $800 \mathrm{mg}$ for at least 1 month prior to study entry. Patients with dyskinesia were excluded. The primary outcome was a change in the total PDQ-8 score. PDQ-8 is a motor and non-motor eight domain questionnaire ranging from 0 (no impairment) to 32 . It is highly correlated with the PDQ-39 assessment tool. Secondary outcome measures were the UPDRS (parts I, II, III and IV) scores and the proportion of patients experiencing wearing off periods. Seventy percent had no fluctuations at baseline and 30\% had non-disabling motor complications (UPDRS part IV score was only 1.3 of a maximum of 23). One hundred and eighty four patients were randomized to either CLE or CL treatment groups in a 1:1 ratio. They found a 1.4 point difference in the PDQ-8 score favoring CLE treatment $(P=0.41)$. UPDRS part II score improved in CLE group $(P=0.032)$ and part III score improved in both groups. The percentage of patients reporting at least one wearing off symptom dropped from $78.5 \%$ to $62 \%$ in the CLE group and from $84.5 \%$ to $61.5 \%$ in the CL group. The difference between groups was not significant. Fourteen patients $(7.6 \%)$ discontinued the study, six in CLE and four in the CL groups. At least one adverse event was reported by $66 \%$ and $56 \%$ of patients in the CLE and CL group respectively. More common adverse events were urine discoloration, nausea, dizziness, constipation, and diarrhea. Mild dyskinesia was reported in 5 patients in the CLE group (5.4\%) and 1 patient in the CL group (1.1\%). ${ }^{43}$ This study failed to show its primary outcome.

The FIRST-STEP study group evaluated the safety, tolerability and efficacy of CLE compared with CL in early PD patients warranting levodopa treatment. It was a 39-week, multicenter, randomized, double-blind, parallel-group study. Eligible patients needed to have UPDRS part II plus part III scores of more than 18 points and H\&Y stage 1-2.5. Patients with more than 5-year disease duration were excluded. Mean age was 65 years, mean disease duration 1.16 years and mean UPDRS (part II and III) score was 34 . Only $2.4 \%$ of patients were on levodopa, $8 \%$ on dopamine agonists, and $34 \%$ on 
other PD medications. They were randomized 1:1 to CLE (25/100/200) mg 3 times daily (tid) or CL (25/100) mg tid. Primary outcome was the change in UPDRS (part II and III) scores. Secondary outcomes were the change in UPDRS subscale scores, PDQ-39 and PDQ-8 scores, H\&Y stage, Schwab and England $(\mathrm{S}+\mathrm{E})$ ADL score, and CGI scores. Treatment success was defined as a reduction of UPDRS total score of more than 8 points. The mean change in total UPDRS score was 10 points in the CLE and 8.5 points in the CL group favoring CLE $(P=0.045)$. Secondary efficacy results showed greater improvement with CLE in UPDRS ADL (part II) subscale by 3 points $(P=0.025), \mathrm{S}+\mathrm{E}$ scores by 5.6 points $(P=0.003)$ and patient CGI $(P=0.047)$. No significant change noted in UPDRS part III (motor score), PDQ-39, PDQ-8 and H\&Y stage. Interestingly, there was no significant change in the incidence of dyskinesia and off-time between groups. Dyskinesia was observed in $11(5.3 \%)$ subjects in the CLE and $16(7.4 \%)$ in the CL group. Twentyfour patients $(11.6 \%)$ in the CLE and eighteen $(8.4 \%)$ in the CL group discontinued due to AE. The incidence of AE was higher in the CLE (82\%) than the CL (60\%) group. Nausea and diarrhea were the most common. ${ }^{44}$ The primary outcome was achieved in this trial.

The STRIDE-PD study evaluated whether CLE would delay the onset of dyskinesia compared with CL in patients with early PD requiring levodopa treatment. In this multicenter study, 747 patients were randomized to either CLE 25/100/200 mg or CL 25/100 mg with dosing every 3.5 hours and up to $400 \mathrm{mg}$ of daily levodopa dose. Treatment duration was variable between 134 and 208 weeks. The primary endpoint was the time to onset of dyskinesia. It was reported that patients treated with CLE had shorter time to dyskinesia and higher incidence of dyskinesia. ${ }^{45}$ Therefore, the study did not achieve its goal.

\section{Cost effectiveness}

In one study conducted in the UK, cost-effectiveness of CLE was compared to standard care in PD patients with wearing-off using the Markov model. The costs and outcomes of both treatments were projected over a period of 10 years. Sensitivity analyses were used to assess the confidence level of the results. CLE produced a reduction in the total 10-year direct cost to society of $£ 10,198$ per patient. The Monte Carlo sensitivity analysis indicated that the likelihood of CLE being either "dominant" (ie, better clinical outcome with lower cost) or more effective at an acceptable cost was $93 \%$ even when all uncertainties associated with the model were taken into account. When compared to standard care, the probability that CLE would be cost-effective to the society as a whole was $77 \% .{ }^{46}$ We should interpret the results of this study with caution as to its ability to be generalized to other countries.

\section{Patient group/population}

The population in the CLE studies consisted mainly of PD patients with early to moderately advanced disease, with non-disabling wearing-off symptoms and mild, if at all, dyskinesia. This is particularly true for the studies that compared CL to CLE, either on direct switch from the former to the latter, ${ }^{41,42}$ or comparing directly the two treatments. ${ }^{43,44}$ The mean disease duration ranged from 1.16 to 9 years. The $\mathrm{H} \& \mathrm{Y}$ stage ranged from 1 (unilateral disease) to 3 (bilateral disease with postural instability). The only study that included more advanced patients was the one that assessed switching from SR-CL to CLE. ${ }^{39}$ This study's population were patients with disease duration of more than 10 years, with $80 \%$ experiencing wearing-off and $47 \%$ dyskinesia at baseline. Those being naïve to entacapone had higher AE and discontinuation rates.

\section{Dosage, administration, and formulation}

CLE tablets are taken orally and are available in different strengths (numbers in mg correspond to carbidopa/levodopa/ entacapone dose, respectively): ${ }^{47}$

$12.5 \mathrm{mg} / 50 \mathrm{mg} / 200 \mathrm{mg}$

$18.75 \mathrm{mg} / 75 \mathrm{mg} / 200 \mathrm{mg}$

$25 \mathrm{mg} / 100 \mathrm{mg} / 200 \mathrm{mg}$

$31.25 \mathrm{mg} / 125 \mathrm{mg} / 200 \mathrm{mg}$

$37.5 \mathrm{mg} / 150 \mathrm{mg} / 200 \mathrm{mg}$

$50 \mathrm{mg} / 200 \mathrm{mg} / 200 \mathrm{mg}$

\section{Place in therapy}

In all the clinical trials so far, CLE exhibits a favorable efficacy, safety and tolerability profile, equivalent to the entacapone studies. However, there was a greater incidence of "dopaminergic" side effects in patients taking CLE compared with CL, with nausea being the most frequent. They were rated as mild and easily manageable in the majority of the cases. The discontinuation rate and incidence of adverse events seem to be more prominent in older patients, naïve to entacapone with more advanced disease, longer disease duration and higher daily levodopa dose. ${ }^{39}$ Although the FIRST-STEP study results showed no statistically different rate of dyskinesia between CLE and CL groups in early PD patients, unpublished data from the STRIDE-PD study 
reported that early PD patients treated with CLE are likely to develop dyskinesia sooner and at a higher rate than the ones treated with CL. ${ }^{44,45}$ Of note is the fact that these are the only long term studies so far.

Efficacy, safety and tolerability outcomes along with the population characteristics suggest that CLE is a safe treatment with a mild side effect profile. It improves quality of life, decreases mean daily "off" time and increases daily "on" time in PD patients with moderately advanced disease and non-disabling "wearing-off" without or with mild dyskinesia. Patients can be switched directly from CL with or without entacapone without having to decrease the levodopa dose. CLE is more convenient for the patients and preferred by the majority of them. There is also evidence in one study that it is feasible to switch from SR-CL to CLE. ${ }^{39}$

CLE cannot be recommended in early PD patients to prevent the development of dyskinesia. It should not be considered in patients with disease duration greater than 10 years, significant wearing-off with troublesome dyskinesia and whose total daily levodopa dose is more than 600 mg. ${ }^{39,45}$

There is no evidence to support a favorable costeffectiveness profile for CLE. The one well conducted study concluded that CLE is cost-effective. ${ }^{46}$ However, we have to take into consideration that this study was conducted in the UK where a national health service is in place. Therefore, its results may not reflect the intricacies of different health care systems.

\section{Disclosures}

Dr Waters receives honoraria for speaking from Teva, Boehringer Ingelheim and Novartis. Dr Poulopoulos reports no conflicts of interest.

\section{References}

1. Cotzias GC, Van Woert MH, Schiffer LM. Aromatic amino acids and modification of parkinsonism. N Engl J Med. 1967;276:374-379.

2. Henchcliffe C, Waters C. Entacapone in the management of Parkinson's disease. Expert Opin Pharmacother. 2002;3(7):957-963.

3. Dunner DL, Brodie HK, Goodwin FK. Plasma DOPA response to levodopa administration in man: effects of a peripheral decarboxylase inhibitor. Clin Pharmacol Ther. 1971;12(2):212-217.

4. Dingemanse J. Catechol-O-methyltransferase inhibitors: clinical potential in the treatment of Parkinson's disease. Drug Dev Res. 1997;42(1):1-25.

5. Nutt JG, Woodward WR, Beckner RM, et al. Effect of peripheral catechol-O-methyltransferase inhibition on the pharmacokinetics and pharmacodynamics of levodopa in parkinsonian patients. Neurology. 1994;44(5):913-919.

6. Ruottinen HM, Rinne UK. Entacapone prolongs levodopa response in a one-month double-blind study in parkinsonian patients with levodopa related fluctuations. J Neurol Neurosurg Psychiatry. 1996;60(1):36-40.
7. Parkinson Study Group. Entacapone improves motor fluctuations in levodopa-treated Parkinson's disease patients. Ann Neurol. 1997;42(5):747-755.

8. Rinne UK, Larsen JP, Siden A, Worm-Petersen J. Entacapone enhances the response to levodopa in parkinsonian patients with motor fluctuations. Nomecomt Study Group. Neurology. 1998;51(5):1309-1314.

9. Poewe W, Deuschl G, Gordin A, Kultalahti ER, Leinonen M. Efficacy and safety of entacapone in Parkinson's disease patients with suboptimal levodopa response: a 6-month randomized placebo-controlled doubleblind study in Germany and Austria (CELOMEN study). Acta Neurol Scand. 2002;105(4):245-255.

10. Brooks DJ, Sagar H. Entacapone is beneficial in both fluctuating and non-fluctuating patients with Parkinson's disease: a randomized, placebo controlled, double blind, six month study. J Neurol Neurosurg Psychiatry. 2003;74(8):1071-1079.

11. Lees AJ. Unresolved issues relating to the shaking palsy on the celebration of James Parkinson's 250th birthday. Mov Disord. 2007;22 Suppl 17:S327-S334.

12. Hirtz D, Thurman DJ, Gwinn-Hardy K, Mohamed M, Chaudhuri A, Zalutsky R. How common are the "common" neurologic disorders? Neurology. 2007;68(5):326-337.

13. Elbaz A, Bower JH, Maraganore DM, et al. Risk tables for parkinsonism and Parkinson's disease. J Clin Epidemiol. 2002;55(1):25-31.

14. Elbaz A, Bower JH, Peterson BJ, et al. Survival study of Parkinson disease in Olmsted County, Minnesota. Arch Neurol. 2003;60(1):91-96.

15. Driver JA, Kurth T, Buring JE, Gaziano JM, Logroscino G. Parkinson disease and risk of mortality: a prospective comorbidity-matched cohort study. Neurology. 2008;70(16):1423-1430.

16. Braak H, Del Tredici K, Rüb U, de Vos RAI, Steur ENHJ, Braak E. Staging of brain pathology related to sporadic Parkinson's disease. Neurobiol Aging. 2003;24(2):197-211.

17. Dickson DW, Fujishiro H, DelleDone A, et al. Evidence that incidental Lewy body disease is pre-symptomatic Parkinson's disease. Acta Neuropathol. 2008;115(4):437-444.

18. Gasser T. Update on the genetics of Parkinson's disease. Mov Disord. 2007;22 Suppl 17:S343-S350.

19. Hirsch EC, Hunot S. Neuroinflammation in Parkinson's disease: a target for neuroprotection? Lancet Neurol. 2009;8(4):382-397.

20. Olanow CW. The pathogenesis of cell death in Parkinson's disease. Mov Disord. 2007;22 Suppl 17:S335-S342.

21. Fahn S, Cohen G. The oxidant stress hypothesis in Parkinson's disease: evidence supporting it. Ann Neurol. 1992;32(6):804-812.

22. Fahn S, Jankovic J. Principles and Practice of Movement Disorders. 1st ed. Philadelphia, PA: Churchill Livingstone Elsevier; 2007.

23. Costello S, Cockburn M, Bronstein J, Zhang X, Ritz B. Parkinson's disease and residential exposure to Maneb and Paraquat from agricultural applications in the central valley of California. Am J Epidemiol. 2009;169(8):919-926.

24. Thacker EL, O'Reilly EJ, Weisskopf MG, et al. Temporal relationship between cigarette smoking and risk of Parkinson disease. Neurology. 2007;68(10):764-768.

25. Hughes AJ, Daniel SE, Kilford L, Lees AJ. Accuracy of clinical diagnosis of idiopathic Parkinson's disease: a clinico-pathological study of 100 cases. J Neurol Neurosurg Psychiatry. 1992;55(3):181-184.

26. Jankovic J. Parkinson's disease: clinical features and diagnosis. J Neurol Neurosurg Psychiatry. 2008;79(4):368-376.

27. Shahed J, Jancovic J. Exploring the relationship between essential tremor and Parkinson's disease. Parkinsonism Relat Disord. 2007;13(2):67-76.

28. Riley D, Lang AE, Blair RD, Birnbaum A, Reid B. Frozen shoulder and other shoulder disturbances in Parkinson's disease. J Neurol Neurosurg Psychiatry. 1989;52(1):63-66.

29. Lim SY, Fox SH, Lang AE. Overview of the extranigral aspects of Parkinson disease. Arch Neurol. 2009;66(2):167-172.

30. Chaudhuri KR, Healy DG, Schapira AHV. Non-motor symptoms of Parkinson's disease: diagnosis and management. Lancet Neurol. 2006;5(3):235-245. 
31. Olanow CW, Stern MB, Sethi K. The scientific and clinical basis for the treatment of Parkinson disease. Neurology. 2009;72(21 Suppl 4): S1-S136.

32. Waters C. Diagnosis and Managements of Parkinson's Disease. 6th ed. New York, NY: Professional Communications, Inc.; 2008.

33. Fahn S. The history of dopamine and levodopa in the treatment of Parkinson's disease. Mov Disord. 2008;23 Suppl 3:S497-S508.

34. The Parkinson Study Group. Levodopa and the progression of Parkinson's disease. N Engl J Med. 2004;351(24):2498-2508.

35. Parkinson Study Group. Pramipexole versus levodopa as initial treatment for Parkinson disease: a randomized controlled trial. JAMA. 2000;284(15):1931-1938.

36. Hauser RA. Levodopa/carbidopa/entacapone (Stalevo). Neurology. 2004;62(1 Supp1 1):S64-S71.

37. Koller W, Guarnieri M, Hubble J, Rabinowicz AL, Silver D. An openlabel evaluation of the tolerability and safety of Stalevo ${ }^{\circledR}$ (carbidopa, levodopa and entacapone) in Parkinson's disease patients experiencing wearing-off. J Neural Transm. 2005;112(2):221-230.

38. Brooks DJ, Agid Y, Eggert K, Widner H, Østergaard K, Holopainen A; TC-INIT Study Group. Treatment of end-of-dose wearing-off in Parkinson's disease: Stalevo ${ }^{\circledR}$ (levodopa/carbidopa/ entacapone) and levodopa/DDCI given in combination with Comtess ${ }^{\mathbb{B}} /$ Comtan $^{\circledR}$ (entacapone) provide equivalent improvements in symptom control superior to that of traditional levodopa/DDCI treatment. Eur Neurol. 2005;53(4):197-202.

39. Lyons KE, Pahwa R. Conversion from sustained release carbidopa/ levodopa to carbidopa/levodopa/entacapone (Stalevo) in Parkinson disease patients. Clin Neuropharmacol. 2006;29(2):73-76.
40. Myllylä V, Haapaniemi T, Kaakkola S. Patient satisfaction with switching to Stalevo: an open-label evaluation in PD patients experiencing wearingoff (Simcom Study). Acta Neurol Scand. 2006;114(3):181-186.

41. Linazasoro G, Kuliseversusky J, Hernández B. Should levodopa dose be reduced when switched to Stalevo? Eur J Neurol. 2008;15(3): 257-261.

42. Boiko AN, Batysheva TT, Minaeva NG, et al. Use of the new levodopa agent Stalevo (levodopa/carbidopa/entacapone) in the treatment of Parkinson's disease in out-patient clinical practice (the START-M open trial). Neurosci Behav Physiol. 2008;38(9):933-936.

43. Fung VSC, Herawati L, Wan Y. Quality of life in early Parkinson's disease treated with levodopa/carbidopa/entacapone. Mov Disord. 2009;24(1):25-31.

44. Hauser RA, Panisset M, Abbruzzese G, Mancione L, Dronamraju N, Kakarieka A. Double-blind trial of levodopa/carbidopa/entacapone versus levodopa/carbidopa in early Parkinson's disease. Mov Disord. 2009;24(4):541-550.

45. Orion.fi/en (homepage on the Internet). Orion Pharmaceuticals Online Press Release. Available from: http://192.49.218.132/en/News-and-media/ Stock-exchange-releases/Archive/1/11/. Accessed on November 10, 2009.

46. Findley LJ, Lees A, Apajasalo M, Pitkänen A, Turunen H. Cost-effectiveness of levodopa/carbidopa/entacapone (Stalevo) compared to standard care in UK Parkinson's disease patients with wearing-off. Curr Med Res Opin. 2005;21(7):1005-1014.

47. Fda.gov (homepage on the Internet). US Food and Drug Administration. Available from: http:/www.accessdata.fda.gov/scripts/cder/ drugsatfda/index.cfm?fuseaction=Search.DrugDetails. Accessed on November 10, 2009.
Core Evidence

\section{Publish your work in this journal}

Core Evidence is an international, peer-reviewed open-access journal evaluating the evidence underlying the potential place in therapy of drugs throughout their development lifecycle from preclinical to postlaunch. The focus of each review is to evaluate the case for a new drug or class in outcome terms in specific indications and patient

\section{Dovepress}

groups. The manuscript management system is completely online and includes a very quick and fair peer-review system, which is all easy to use. Visit http://www.dovepress.com/testimonials.php to read real quotes from published authors. 